\title{
Robotic Surgery for Obstructive Sleep Apnea
}

\author{
Claudio Vicini · Filippo Montevecchi • \\ J. Scott Magnuson
}

Published online: 19 June 2013

(C) Springer Science+Business Media New York 2013

\begin{abstract}
Nocturnal upper airway collapse often involves obstruction at the level of the tongue base. A number of surgical procedures have been developed in recent years to address this area in patients non-compliant with CPAP therapy. This paper outlines a novel way to treat obstructive sleep apnea related to lingual obstruction, utilizing the da Vinci $^{\circledR}$ robotic surgical system. This technique offers significant potential advantages over other established approaches, and should be included in the surgical armamentarium of sleep surgeons.
\end{abstract}

Keywords TORS $\cdot$ Apnea $\cdot$ Tongue base $\cdot$ Sleep medicine

\section{Introduction}

Nocturnal upper airway collapse often involves obstruction at the level of the tongue base. In patients with obstructive sleep apnea (OSA) who are non-compliant with CPAP therapy a number of surgical procedures have been developed in recent years to address the area of the base of

C. Vicini · F. Montevecchi

Otolaryngology-Head and Neck and Oral Surgery Unit, Special Surgery Department, Morgagni Pierantoni Hospital, University of Pavia in Forlì, Via Carlo Forlanini, 34, 47100 Forlì, Italy e-mail: claudio@claudiovicini.com

F. Montevecchi

e-mail: filo651@hotmail.com

J. Scott Magnuson ( $\square)$

Head and Neck Surgery, Center of Florida, Florida Hospital, Celebration Health, 410 Celebration Place,

Suite 305, Celebration, FL 34747, USA

e-mail: scott.magnuson@flhosp.org tongue. These include hyolingual advancement, tongue suture suspension, and various lingual resection techniques [1-6].

Typically, conventional tongue base resection is performed either via a transcervical approach or transorally with the aid of an endoscope for visualization [7-9]. These approaches each has significant potential limitations. The open techniques are technically difficult and morbid, and the transoral endoscopic approaches are hampered by relatively poor exposure and limited, non-articulated instrumentation.

\section{Definitions}

Collectively, trans oral robotic surgery (TORS) procedures involve the precise excision of oral, pharyngeal and laryngeal tissue with a surgical robot. The robot facilitates these procedures with its articulated instruments and 3-dimension, high definition camera. TORS was pioneered by Weinstein and O'Malley at the University of Pennsylvania as a minimally invasive technique for treatment of oropharyngeal cancers [10]. FDA approval for a number of adult head and neck indications was granted in December 2009 [11]. TORS has since become adopted worldwide and is considered by many practitioners to be the most effective and reproducible technique available for upper aerodigestive tract surgery. At face value, the outcomes of TORS appear equivalent to traditional transoral surgeries. However, numerous peer reviewed articles have shown that TORS allows the surgeon to perform procedures that otherwise could be done only through an open approach, but with less morbidity and improved quality of life (Fig. 1).

Performing tongue base reduction (TBR) with the TORS approach allows for removal of a part of the base of the 


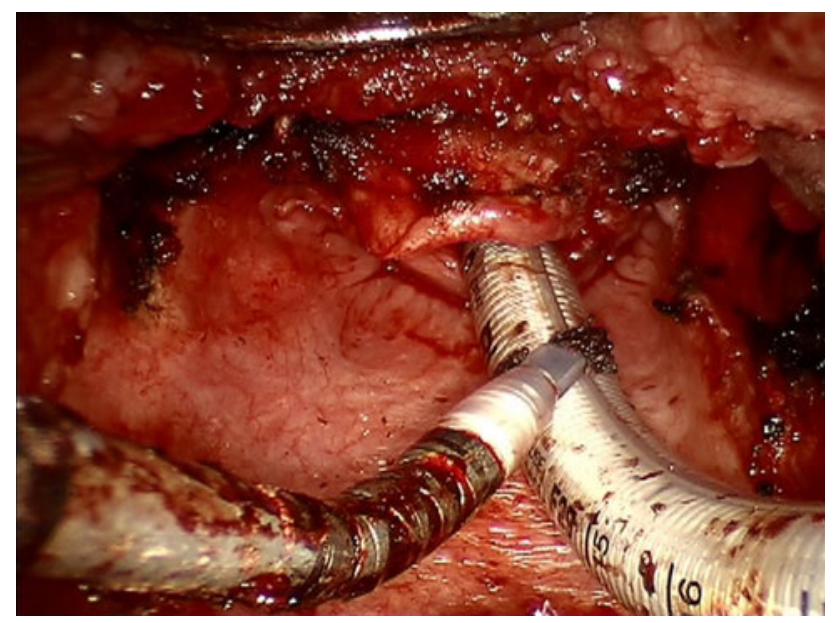

Fig. 1 Surgical field after tongue base reduction and before supraglottoplasty

tongue from the foramen cecum to the vallecula. Note that in the context of sleep surgery, the term "reduction" is preferred to "resection". TBR can include removal of lingual tonsillar tissue, tongue base musculature, or both. The amount of tissue resected can vary from several $\mathrm{ml}$ to more than $50 \mathrm{ml}$. This is determined by each individual patient's anatomy and degree of prolapse during sleep.

Supraglottoplasty (SGP) refers to the adjunctive surgical management of collapsible epiglottic, arytenoid and/or aryepiglottic tissue. The suffix "plasty" includes a broad range of surgical maneuvers. Again, the specific manipulation utilized is tailored to the presenting pathology.

\section{Indications}

TORS TBR SGP can be used for appropriate cases of clinically significant OSA [12]. Most patients treated with this surgery have polysomnographic evidence of at least moderate to severe OSA (respiratory disturbance index $\geq 20$ ) and excessive daytime somnolence (as documented by an Epworth Sleepiness Scale score $>10$ ). Ideal candidates for TORS intervention have significant obstruction at the tongue base (with a Cormack and Lehane grade of $>2$ ) and/or prolapse of adjacent supraglottic tissue. This is best determined by awake supine endoscopic exam or by druginduced sleep endoscopy (DISE).

TORS tongue base surgery can be offered as a primary procedure to patients non-compliant with CPAP or as a secondary procedure after previous surgery failed to resolve the OSA. As per the surgeon's judgment associated nasal obstruction and excessive palate and tonsil tissue may be treated concurrent with TORS surgery. However, it has been reported that simultaneous multilevel surgery can increase the potential for postoperative morbidity [14].
Surgical procedures that have been performed concurrently with TORS TBR SGP include septal and turbinate surgery, as well as a number of variations of palate surgery, such as traditional uvulopalatopharyngoplasty, expansion sphincteroplasty, and Z-palatoplasty $[15,16]$. Concurrent pharyngeal surgery may be carried out conventionally or robotically, according to the surgeon's preference and/or experience.

\section{Contraindications}

There are several important contraindications to TORS TBR SGP. Surgery should not be offered to patients who are successfully treated with non-operative management. It is well accepted that OSA surgery should not be offered to patients who are tolerant of and compliant with CPAP therapy [13]. Other contraindications include comorbidities that result in an ASA score $>2$ or which would suggest that sleep surgery not be performed, such as significant or unstable cardiovascular disease, a history of a cerebrovascular accident, progressive neuromuscular disease, need for anticoagulation, or significant psychological instability.

As with any surgery, adequate exposure is crucial. For TORS, surgeons must verify that a potential patient's anatomy will allow for sufficient exposure intraoperatively. Signs of potentially difficult exposure include significant retrognathia, an inability to hyperextend the neck, an interincisive distance $<2.5 \mathrm{~cm}$, and trismus. Significant micrognathia and macroglossia (with high modified MallampatiFriedman scores) can limit exposure for TORS, though we have operated on many such patients successfully.

\section{Preoperative Work-Up}

The preoperative workup is the same as what has been described for OSA surgery in general, including a polysomnographic evaluation, thorough sleep history, assessment of general medical health, and a comprehensive upper airway examination with awake fiberoptic endoscopy. In addition to the general ENT exam, this assessment should include notation of body mass index, neck circumference, tonsil size (grade 0-IV), modified Mallampati-Friedman Scoring (I-IV), and Cormack and Lehane Scoring. Documentation of somnolence and sleep quality can include notation of the Epworth Sleepiness Scale score and results of any of the standardized subjective quality of life questionnaires (SF36, Stanford Sleepiness Scale, Beck Depression Index, etc.). Radiographic imaging is not essential, but when indicated can include panoramic radiography and lateral cephalometry, computerized tomography, and magnetic resonance imaging [17]. Though not 
routinely utilized by most surgeons before OSA surgery, neck CT or MRI can help in select cases to better define the pattern of soft tissue collapse and the relative mass of the obstructive lingual tissue. Imaging can also assist by demonstrating if tissue is primarily lymphatic (lingual tonsillar tissue), muscular, or both. In addition, druginduced fiberoptic sleep endoscopy (DISE) has been found to be a helpful determinant in site of obstruction determination $[18,19 \bullet, 20,21]$. Though DISE is not yet a routine part of the preoperative evaluation of OSA patients in the US, there is a long track record of this procedure in Europe, and its adoption in the US appears to be growing.

\section{Anesthesia}

TORS TBR SGP is performed under general anesthesia [22]. The anesthesia and surgical team must be prepared for a difficult intubation, which could require the use of specialized instrumentation, such as a fiberoptic endoscope, GlideScope, or light wand. An extended anesthesia respiratory circuit is necessary, as the anesthesiologist is stationed at the foot of the bed. This allows for the surgical assistant to sit at the head of the bed without interference and allows for proper positioning of the robotic carts. They are placed along the sides of the patient's head as described for other TORS procedures. In Europe it is more customary to perform a tracheostomy immediately after intubation for lingual TORS surgery [23, 24]. This is not routinely advocated in the US and the surgery is done after either orotracheal or nasotracheal intubation with a small caliber endotracheal tube. Intravenous steroids are given to minimize lingual edema and for nausea prevention and intravenous broad-spectrum antibiotics are infused preoperatively per hospital protocol or surgeon preference.

\section{Instrumentation}

This surgery is performed with the da Vinci ${ }^{\circledR}$ Surgical System (Intuitive Surgical, Sunnyvale, CA). TORS requires either the S HD or the Si da Vinci ${ }^{\circledR}$ models; the Standard (or S) system is not sufficient for these procedures. This robotic equipment includes:

- High definition camera. A 0 and $30^{\circ}$ camera is available with the robotic system. Both the 12 and the $8 \mathrm{~mm}$ diameter scope have excellent optics and allow for adequate working space in the mouth. They provide magnification up to $10 \times$, resulting in a clear 3D image, with easy identification of vessels and nerves.

- Two $5 \mathrm{~mm}$ articulated Endo Wrist ${ }^{\circledR}$ instrument arms, one placed on either side of the camera. A grasper is placed on one arm-usually a $5 \mathrm{~mm}$ Maryland forceps is used-and a spatula tip monopolar cautery in the other arm. A compatible laser fiber can be used in lieu of the cautery and is preferred by some surgeons.

Though TORS surgery does not require much additional equipment beyond the robotic tools listed above, there are several other important instruments, which should be readily available on the operating room back table. It is best to have all of these instruments open and readily accessible. These include:

- Mouth gags: Two commonly used mouth gags include the Davis-Meyer ${ }^{\circledR}$ gag (Karl Storz America) and the Feyh-Kastenbauer-Weinstein-O'Malley retractor (Gyrus ACMI, Germany). The Davis-Meyer system includes multiple tongue blades of different lengths, each with integrated suction tubes for smoke evacuation, and is usually sufficient for the procedure. A small sized blade is usually best, especially in the initial TBR dissection. The FKWO gag has integrated adjustable cheek retractors but no suction ports on its various sized tongue blades; this retractor is sometimes superior to the Davis-Meyer system for the SGP portion of the surgery.

- Headlight: A headlight is used when placing the mouth gag to assure adequate exposure.

- The robotic monopolar cautery is usually sufficient for hemostasis, although a vessel clip applier and an insulated bipolar forceps should be readily available. A tonsillectomy type suction cautery device is also sometimes needed.

- The bedside assistant may use a small diameter Yankauer suction device to suction blood and smoke from the surgical field. The suction device may also be used as a retractor.

- A few appropriate length forceps, hemostats, scissors, and other basic soft tissue instruments are occasionally needed.

\section{Surgical Team}

The bedside TORS team is composed of:

- The primary surgeon is seated at the robotic console. This console is situated in close proximity to the OR table, usually off to one side but within view of the operative site.

- The assistant (which may be a second surgeon) is seated at the head of the patient. The assistant retracts tissue, evacuates blood and smoke, adjusts the robotic arms 
and camera as needed to avoid robotic arm collisions, and assists with hemostasis via bipolar electrocoagulation or with use of vessel clips. An integrated microphone-loudspeaker system is built into the robotic system to improve communication between the primary surgeon and assistant.

- A surgical scrub technician, who aids in passing instruments and providing equipment is needed.

\section{Surgical Steps}

\section{Tongue Base Reduction (TBR)}

TBR is based on the principles of TORS tongue base resection described in 2006 by O'Malley et al. [25••], and enlarges the anteroposterior airway dimension at the level of the tongue base. This reduction usually extends rostrally from the foramen cecum level to the vallecula; the SGP extension of the procedure can include vallecular mucosa and/or part of the epiglottis as necessary. The lateral oropharyngeal dimensions are not augmented with this surgery, and are better addressed by tonsillectomy with one of the variations of UPPP or lateral pharyngoplasty. As there can be considerable variation in anatomy between patients, the goal is not to remove a predetermined volume of tongue base but to improve the Cormack and Lehane grade from III or IV to at grade II or better [26]. Though it is safest to limit the tissue resection to the superficial layer of lingual lymphoid tissue, most cases require extension of the dissection into the lingual musculature. This deeper dissection can lead to exposure of the lingual artery and its dorsal branches, the hypoglossal nerve, and the lingual nerve. Though the volume of excised tissue is generally in the $14-20 \mathrm{ml}$ range, this can vary from as little as $7 \mathrm{ml}$ to as much as $50 \mathrm{ml}$. The procedure is divided into several standardized steps, outlined below, and usually takes about 30 min to complete [27••, 28••, 29].

\section{Exposure}

The intubated patient is placed supine, the neck is flexed, and the head is hyperextended and placed in the so-called "sniffing position". If a planned tracheotomy is to be performed, it is done at this point. An upper dental arch tooth guard is placed to protect the area from trauma from the robotic camera and instrument arms. The tongue is pulled anteriorly with stay sutures and by using one of the two mouth gags. This maneuver can result in compression of the ventral tongue against the lower incisors. Consequently, consideration should also be given to use of a lower tooth guard to prevent any injury. The tongue base is then exposed with the appropriate mouth gag. The length of the tongue blade and amount of tongue retraction is adjusted so that the distal end of the blade ends just rostral to the superior limit of the planned resection in the midline (usually around the foramen cecum). It is not unusual at this point for the epiglottis to be obscured by the tongue base. If this is the case, it will come into view as the tongue reduction progresses. The robot is docked as per normal TORS protocol.

\section{Lingual Tonsillectomy}

(a) For the right side of the lingual tonsillectomy the Maryland forceps is placed in the left arm and the spatula tip cautery in the right arm. For the left side the instruments are switched.

(b) The $30^{\circ}$ endoscope is positioned face up. The camera is set at relatively low magnification and wide-angle view to give a panoramic view of the tongue base at the beginning of the case. As the case progresses the magnification can be raised. The incision begins in the midline from the rostral end of the planned resection to the vallecula. The foramen cecum is a good landmark for the upper extent of this incision, as it is distal to the circumvallate papillae and is in the midline. This incision splits the lingual tonsillar tissue in the midline and extends down to the junction between lymphoid tissue and the underlying muscle.

(c) The borders of the right lingual tonsil are identified and marked with cautery to outline the resection margins. This extends superiorly from the sulcus terminalis, laterally from the amygalo-glossal sulcus, and inferiorly to the glosso-epiglottic sulcus. If the lingual tonsillar tissue is bulky enough that these landmarks are not visible, the central tongue base can first be debulked.

(d) The target tissue is then resected, with the deep plane at the lympho-muscular junction. This can usually be done in an en bloc fashion with minimal blood loss. The bedside assistant can facilitate this resection by using a suction or retracting device for counter traction of the tissue. The inferior limit of the surgical bed can be recognized by the bluish color of the vallecular mucosa. A number of small vessel branches from the lingual artery will be encountered and usually are taken care of easily with cautery. Larger vessels may be clipped as needed. Visualization of these vessels is improved by increasing the scope magnification.

(e) Additional tissue is removed as needed to assure a Cormack and Lehane grade of 2 or better. A minimal overall volume of $7 \mathrm{ml}$ is recommended for alleviating obstruction. This may include muscle, with the following caveats, based on cadaveric dissections [29-31]: 
- It is usually safe to remove a layer of muscle $\leq 10 \mathrm{~mm}$ within the entire tongue base.

- An additional $5 \mathrm{~mm}$ thick strip of muscle may generally be safely removed in the tongue base within $5 \mathrm{~mm}$ of the midline without encountering the hypoglossal nerves.

- This additional midline muscle resection should be done carefully and under sufficiently high magnification. It is of paramount importance to use the tongue base midline as the point of reference to minimize the risk to the hypoglossal nerve, lingual artery, and lingual neural branches. Because of inherent anatomic variability and tissue distortion from tongue retraction and mouth gag placement, precise localization of these structures is not possible.

\section{Supraglottoplasty (SGP)}

This optional step is performed at the same operative setting immediately after completion of the TBR. SGP is designed to ameliorate the inward inspiratory collapse of floppy and/or redundant epiglottic, aryepiglottic, and arytenoid mucosa $[25 \bullet \bullet, 27 \bullet \bullet, 32]$. This can include any or all of the following maneuvers and can usually be performed in 5 min or less:

- Resection of excessive tissue.

- Tissue removal to promote scarring and retraction.

- Use of sutures to increase tissue stability.

- Sectioning of tissue to release overly short ligaments.

The two most common SGP techniques performed are the vertical midline suprahyoid epiglottic split and the horizontal epiglottic transection.

\section{Vertical Midline Supra-Hyoid Epiglottic Split}

The epiglottis is incised in the midline, following the medial glosso-epiglottic fold. This split begins at the epiglottic tip and extends caudally to a level $\geq 5 \mathrm{~mm}$ cephalad to the vallecula. A sufficient amount of epiglottic height should be left to minimize the chance for aspiration.

\section{Horizontal Epiglottic Transection}

The inferior extent of the midline epiglottic split is extended laterally on both sides. The plane of resection is cephalad to the pharyngo-epiglottic folds to minimize the chance for aspiration and to avoid bleeding from branches of the superior laryngeal vessels. In addition to stabilizing this supraglottic tissue, the secondary intention healing in the vallecula and adjacent areas from this procedure results in anterior advancement of the epiglottis. Caution has to be used to avoid overly aggressive resection of vallecular and epiglottic tissue as this can result in aspiration from fixation of the epiglottis to the tongue base.

\section{Case Conclusion and Postoperative Care}

The wound is inspected carefully to verify adequate hemostasis. Cautery or vessel clips are used as needed for persistent bleeding. A hemostatic agent can be applied to the exposed tissue in a thin layer. Some clinicians feel this may also help with postoperative discomfort.

The tongue and airway are inspected for edema. If the patient has not undergone a tracheostomy the patient is assessed for extubation under observation in the OR. Overnight stay with continued intubation can be considered per surgeon's preference if necessary.

Consideration should be given to placement of a nasogastric (NG) feeding tube, and some centers routinely use NG tubes for all TORS patients in the immediate postoperative period. This is per the surgeon's preference.

Given the OSA, airway edema, and the potential for bleeding, it is advised that initial postoperative observation be undertaken in the appropriate monitored setting. In most institutions this would be the intensive care unit (or equivalent) rather than the typical postoperative hospital ward. Continuous pulse-oximetry is recommended.

The patient should have suction available at all times at the bedside. The patient should be watched closely for bleeding, as there is a large area of exposed tissue at the surgical site which must undergo healing by secondary intention. As is the case with all such pharyngeal surgical defects, such as a "simple" tonsillectomy, the risk of postoperative bleeding has a bimodal distribution, with one peak within hours of surgery and the second approximately 7-10 days after surgery.

Postoperative intravenous steroids can help with nausea, airway edema, and pain from the inflammatory response. It is advised to continue oral steroids post-discharge on a tapering dose, as some patients experience a significant crescendo of pain up to a week postoperatively. This is likely due to hyperinflammation or dissolution of the protective fibrinous exudative coating over the surgical site. In addition to steroid use, narcotics are used as needed, with close observation of respiratory rate and level of consciousness.

The length of stay can vary considerably depending upon a number of variables and surgeon comfort level. Tracheostomy by itself necessitates a multi-day hospital stay. Other determinants include the presence or absence of other concurrent OSA procedures (UPPP, e.g.), general patient health, pain control, and swallowing ability. 
Table 1 Complications TORS for OSA (unpublished data from 75 consecutive patients)

\begin{tabular}{lc}
\hline Operative complications & Percentage \\
\hline Transient hypogeusia & 18.30 \\
Intra/post operative transient pharyngeal edema & 3.16 \\
Late self limiting bleeding & 2.50 \\
Teeth injury & 0.0 \\
Pharyngeal wall penetration & 0.0 \\
Intraoperative bleeding & 0.0 \\
Revision surgery & 0.0 \\
Death & 0.0 \\
Conversion to open & 0.0 \\
\hline
\end{tabular}

Patients are followed closely after discharge. Diet is normalized as healing progresses, and formal therapist directed swallowing therapy is rarely needed $(<10 \%$ of cases). Postoperative polysomnography is performed once healing is complete, usually at least 3-6 months afterwards.

\section{Complications}

The largest series of patients to undergo TORS for OSA has been performed by Vicini et al. [28]. The most common operative complications include hypogeusia, transient pharyngeal edema and limited bleeding (Table 1). The pressure from the tongue blade attached to the mouth gag may cause temporary injury to the lingual nerve resulting in numbness that typically resolves in 1-4 weeks. Pharyngeal edema may also occur as a result of the pressure from the tongue blade and heat generated from the monopolar cautery. Both of these complications are directly related to the length of the operation and amount of time the patient is kept in suspension. Patients in suspension for less than $45 \mathrm{~min}$ are less likely to develop these complications.

Late self limiting bleeding is uncommon and is reported to be $2.5 \%$. More severe complications (tooth injury, pharyngeal wall penetration, intraoperative bleeding requiring open control of vessels, and death) have not been reported.

\section{Results}

The series reported by Vicini et al. showed an improvement from a mean preoperative AHI 36.3 to a postoperative AHI 16.4. Fifteen of the 20 patients in this series had a postoperative AHI less than 20 and only two patients had an AHI greater than 30. Improvements were also seen in Epworth Sleepiness Scale, lowest oxygen saturation and overall patient satisfaction. All improvements were found to be statistically significant.

\section{Conclusion}

TORS for OSA allows for tongue base resection with several advantages potential advantages over other tongue base resection procedures. This technique appears to be safe and effective. Additional studies, including comparison with other techniques are needed to assess the proper role of TORS in sleep surgery.

Compliance with Ethics Guidelines This article does not contain any studies with human or animal subjects performed by any of the authors.

Disclosure J. Scott Magnuson is a paid consultant, speaker, and instructor for Intuitive Surgical, Inc. Claudio Vicini and Filippo Montevecchi declare that they have no conflict of interest.

\section{References}

Papers of particular interest, published recently, have been highlighted as:

- Of importance

•- Of major importance

1. Friedman M, Soans R, Gurpinar B, Lin HC, Joseph N. Evaluation of submucosal minimally invasive lingual excision technique for treatment of obstructive sleep apnea/hypopnea syndrome. Otolaryngol Head Neck Surg. 2008;139(3):378-84.

2. Fujita S, Woodson BT, Clark JL, Wittig R. Laser midline glossectomy as a treatment for obstructive sleep apnea. Laryngoscope. 1991;101(8):805-9.

3. Maturo SC, Mair EA. Coblation lingual tonsillectomy. Otolaryngol Head Neck Surg. 2006;135(3):487-8.

4. Michelson SA. Radiofrequency tissue volume reduction of the tongue. In: Terris DJ, Goode RL, editors. Surgical management of sleep apnoea and snoring. New York: Taylor \& Francis; 2005.

5. Blumen MB, Coquille F, Rocchicioli C, Mellot F, Chabolle F. Radiofrequency tongue reduction through a cervical approach: a pilot study. Laryngoscope. 2006;116(10):1887-93.

6. Woodson BT. Innovative technique for lingual tonsillectomy and midline posterior glossectomy for obstructive sleep apnea. Oper Tech Otolaryngol Head Neck Surg. 2007;18:20-8.

7. Chabolle F, Wagner I, Blumen MB, et al. Tongue base reduction with hyoepiglottoplasty: a treatment for severe obstructive sleep apnea. Laryngoscope. 1999;109(8):1273-80.

8. Sorrenti G, Piccin O, Mondini S, Ceroni AR. One-phase management of severe obstructive sleep apnea: tongue base reduction with hyoepiglottoplasty plus uvulopalatopharyngoplasty. Otolaryngol Head Neck Surg. 2006;135(6):906-10.

9. Vicini C. Tongue-base reduction with hyoepiglottoplasty (TBRHE di chabolle). In: Vicini C, editor. Chirurgia della roncopatia. 1st ed. Lucca: Eureka; 2007. p. 253-9.

10. O’Malley BW Jr, Weinstein GS, Snyder W, Hockstein NG. Transoral robotic surgery (TORS) for base of tongue neoplasms. Laryngoscope. 2006;116(8):1465-72.

11. Weinstein GS, O’Malley BW Jr, Magnuson JS, Carroll WR, Olsen KD, Daio L, Moore EJ, Holsinger FC. Transoral robotic surgery: a multicenter study to assess feasibility, safety, and surgical margins. Laryngoscope. 2012;122(8):1701-7. doi:10.1002/lary.23294. 
12. Young T, Peppard PE, Gottlieb DJ. Epidemiology of obstructive sleep apnea: a population health perspective. Am J Respir Crit Care Med. 2002;165(9):1217-39.

13. Giles TL, Lasserson TJ, Smith BH, White J, Wright J, Cates CJ. Continuous positive airways pressure for obstructive sleep apnoea in adults. Cochrane Database Syst Rev. 2006;19(3):CD001106.

14. Sundaram S, Bridgman SA, Lim J, Lasserson TJ. Surgery for obstructive sleep apnoea. Cochrane Database Syst Rev. 2005;19(4):CD001004.

15. Cahali MB, Formigoni GG, Gebrim EM, Miziara ID. Lateral pharyngoplasty versus uvulopalatopharyngoplasty: a clinical, polysomnographic and computed tomography measurement comparison. Sleep. 2004;27(5):942-50.

16. Pang KP, Woodson BT. Expansion sphincter pharyngoplasty: a new technique for the treatment of obstructive sleep apnea. Otolaryngol Head Neck Surg. 2007;137(1):110-4.

17. Barkdull GC, Kohl CA, Patel M, Davidson TM. Computed tomography imaging of patients with obstructive sleep apnea. Laryngoscope. 2008;118(8):1486-92.

18. Abdullah VJ, Van Hasselt CA. Video sleep nasoendoscopy. In: Terris DJ, Goode RL, editors. Surgical management of sleep apnoea and snoring. 1st ed. New York: Taylor \& Francis; 2005.

19. - Campanini A, Canzi P, De Vito A, Dallan I, Montevecchi F, Vicini C. Awake versus sleep endoscopy: personal experience in 250 OSAHS patients. Acta Otorhinolaryngol Ital. 2010;30(2):73-7. 250 patients underwent awake and sleep endoscopy. Sleep endoscopy was useful in determining the site of obstruction.

20. Pringle MB, Croft CB. A comparison of sleep nasoendopscopy and Muller manoeuvre. Clin Otolaryngol. 1991;16:559-62.

21. Kezirian EJ. Nonresponders to pharyngeal surgery for obstructive sleep apnea: insights from drug-induced sleep endoscopy. Laryngoscope. 2011;121(6):1320-6. doi:10.1002/lary.21749.

22. Hillman DR, Loadsman JA, Platt PR, Eastwood PR. Obstructive sleep apnoea and anaesthesia. Sleep Med Rev. 2004;8(6):459-71.

23. Campanini A, De Vito A, Frassineti S, Vicini C. Temporary tracheotomy in the surgical treatment of obstructive sleep apnea syndrome: personal experience. Acta Otorhinolaryngol Ital. 2003;23(6):474-8

24. Sun H, Lou W, Wang L, Wu Y. Clinical significance of preoperative tracheotomy in preventing perioperative OSAHS severe complications. Lin Chuang Er Bi Yan Hou Ke Za Zhi. 2005; 19(9):394-5.

25. •• Vicini C, Montevecchi F, Tenti G, Canzi P, Dallan I, Tod H. Huntley. Transoral robotic surgery: tongue base reduction and supraglottoplasty for obstructive sleep apnea. Operative Technique in Otolaryngology. 2012;23(1): 45-7. TORS (transoral robotic surgery) tongue base reduction technique described for patients with obstructive sleep apnea (OSA).

26. Cormack RS, Lehane J. Difficult tracheal intubation in obstetrics. Anaesthesia. 1984;39:1105-11.

27. • Vicini C, Dallan I, Canzi P, Frassineti S, La Pietra MG, Montevecchi F. Transoral robotic tongue base resection in obstructive sleep apnoea-hypopnoea syndrome: a preliminary report.ORL J Otorhinolaryngol Relat Spec. 2010;72(1):22-7. First paper to describe surgical outcomes for patients with OSA who underwent TORS with tongue base reduction.

28. • Vicini C, Dallan I, Canzi P, Frassineti S, Nacci A, Seccia V, Panicucci E, Grazia La Pietra M, Montevecchi F, Tschabitscher M. Transoral robotic surgery of the tongue base in obstructive sleep apnea-hypopnea syndrome: anatomic considerations and clinical experience. Head Neck. 2012;34(1):15-22. doi: 10.1002/hed.21691. This paper describes the pertinent anatomy of the tongue base from a transoral view for surgeons performing TORS.

29. Vicini C, Montevecchi F, Dallan I, Canzi P, Tenti G. Transoral robotic geniohyoidpexy as an additional step of transoral robotic tongue base reduction and supraglottoplasty: feasibility in a cadaver model. ORL J Otorhinolaryngol Relat Spec. 2011; 73(3): $147-50$.

30. Sequert C, Lestang P, Baglin AC, Wagner I, Ferron JM, Chabolle F. Hypoglossal nerve in its intralingual trajectory: anatomy and clinical implications. Ann Otolaryngol Chir Cervicofac. 1999; 116(4):207-17.

31. Lauretano AM, Li KK, Caradonna DS, Khosta RK, Fried MP. Anatomic location of the tongue base neurovascular bundle. Laryngoscope. 1997;107(8):1057-9.

32. Golz A, Goldenberg D, Westerman ST, Catalfumo FJ, Netzer A, Westerman LM, Joachims HZ. Laser partial epiglottidectomy as a treatment for obstructive sleep apnea and laryngomalacia. Ann Otol Rhinol Laryngol. 2000;109(12):1140-5. 